\title{
Políticas estatales y transformación urbana: ¿hacia un proceso de gentrificación en Valparaíso, Chile?
}

Carlos Vergara-Constela. Universitat Autònoma de Barcelona, Bellaterra, España. Antònia Casellas. Universitat Autònoma de Barcelona, Bellaterra, Espańa.

RESUMEN | A través de un enfoque metodológico mixto, el presente estudio analiza la transformación de la ciudad de Valparaíso (Chile) a partir de la década de 2000, vinculando las características del proceso y los agentes y políticas de renovación urbana, con los efectos en la morfología urbana resultantes de la denominación patrimonial Unesco. El análisis permite identificar al Estado chileno como agente clave en la renovación urbana a través de su papel de liderazgo en la promoción patrimonial, el turismo y la ampliación del mercado universitario. Estas estrategias de crecimiento apuntan a un emergente proceso de gentrificación.

PALABRAS CLAVE | políticas urbanas, Estado, patrimonio, turismo, gentrificación.

ABSTRACT | This study analyses the transformation of the city of Valparaiso (Chile) from the 2000's, through a mixed methodological approach, linking the characteristics of urban renewal process, agents and policies to the effects in urban morphology resulting from the UNESCO heritage designation. The analysis identifies the Chilean State as a key player in urban renewal through its leadership role in promoting heritage, tourism, and the expansion of the university market. These growth strategies point to an emerging process of gentrification.

KEY WORDS | urban policies, State, heritage, tourism, gentrification. 


\section{Introducción}

Entre las transformaciones que más han impactado las ciudades latinoamericanas en las últimas décadas se incluyen la pérdida de peso del sector industrial, la expansión urbana de carácter metropolitano y el deterioro de las zonas céntricas urbanas. En Latinoamérica esto ha ido acompańado de la última fase de modernización capitalista, como un proceso lento y poco advertido, y con dinámicas distintas a aquellas de los países europeos (De Mattos, 2006). Una de las especificidades más relevantes en la región tiene relación con los mecanismos de revaloración patrimonial de los centros históricos impulsados por el Estado y/o gobiernos locales, generalmente acompañados de planes de renovación urbana (Carrión, 2007). Como Michael Pacione (1990) afirma, los centros históricos de las ciudades se encuentran prácticamente sujetos a procesos de constante renovación, siendo uno de los principales motivos la búsqueda de dinamización económica a través del fomento de las actividades culturales y turísticas. A esta estrategia de renovación urbana, en algunas ciudades latinoamericanas -como en el caso de Valparaíso (Chile) - se le ha sumado una estrategia de desarrollo económico y urbanístico sustentada en la expansión de centros universitarios.

La trasformación urbana de los centros urbanos degradados va acompañada a menudo de procesos de gentrificación (Beauregard, 1985; Lees, Slater \& Wyly, 2007). Enmarcada en procesos paralelos de suburbanización y revaloración de espacios centrales, la gentrificación da cuenta de operaciones de reinversión y fijación de capital en espacios determinados, las cuales se traducen en el desplazamiento de habitantes de menores niveles socioeconómicos por otros con mayores ingresos y/o capacidad de consumo. Estos desarrollos son identificables a través de transformaciones de las actividades comerciales y del paisaje urbano predominante en las zonas afectadas (Janoschka, Sequera \& Salinas, 2013).

En este marco, el presente artículo realiza un aporte al cuerpo empírico de los estudios urbanos sobre las políticas de restructuración urbana en Latinoamérica, a partir de un análisis de las estrategias patrimoniales de expansión turística y oferta universitaria aplicadas en el caso de Valparaíso. El estudio se propone contribuir al debate generado por los agentes urbanos provenientes de la sociedad civil de esa ciudad, los cuales han tenido poco margen de actuación, al encontrarse en una posición dominada por el juego de fuerzas políticas y económicas de carácter expansivo (González, 2011).

En términos metodológicos, el artículo se nutre de información cualitativa y cuantitativa, incluyendo el análisis de documentos oficiales de los planes y programas emanados del Plan Valparaíso, como el Programa de Recuperación y Desarrollo Urbano de Valparaíso (PRDUv), el Plan RUMbo (Plan Estratégico para el Destino Turístico Valparaíso), el programa "Estudia en Valparaíso", más los Subsidios de Rehabilitación Patrimonial (Ministerio de Vivienda y Urbanismo, MINVu, y Corporación de Fomento de la Producción, Corfo), a los que se agrega el documento madre de postulación a la Unesco (Organización de las Naciones Unidas para la Educación, la Ciencia y la Cultura) compilado por el Consejo Nacional de Monumentos Históricos ( $\mathrm{CNMH})$, e instrumentos de planificación urbana, como el 
Plan Regulador Comunal. Este análisis se complementa con información primaria y secundaria. Entre las primeras se cuentan observaciones sistemáticas y recopilación de abundante material visual. Entre las segundas, una base de datos de uso de suelo (roles de cobro del Servicio de Impuestos Internos), estadísticas sobre estudiantes universitarios, inversión del Programa de Recuperación y Desarrollo Urbano de Valparaíso y estadísticas censales.

Tomando en consideración el objetivo de estudio, en primer lugar se examina conceptualmente el rol estatal en los procesos de reestructuración urbana y la incidencia del Estado chileno en esos procesos, a través de políticas urbanas de corte proempresarial, para, a continuación, vincular teóricamente el concepto de gentrificación con las políticas urbanas ligadas al pro empresarialismo urbano. Seguidamente se aborda la noción de gentrificación simbólica, entendida como producto de la instalación de procesos de renovación urbana basados en el turismo y la cultura. En el estudio de caso se analizan las políticas urbanas iniciadas en 2002 en Valparaíso, examinándose sus efectos en la morfología urbana y en la creación de un espacio predominantemente turístico en los cerros Alegre y Concepción. Se concluye con algunas reflexiones sobre las condiciones de transformación urbana y sus implicaciones morfológicas y sociales.

\section{El urbanismo proempresarial como estrategia}

Siguiendo a Harvey (1989), se debe comprender el urbanismo proempresarial como una forma de gobernanza urbana que se ajusta a los requerimientos de los gobiernos nacionales y/o locales en el marco de modelos de acumulación flexible de capital, dentro de un esquema de reestructuración económica. En un contexto de competencia entre ciudades por la atracción de capitales e inversiones privadas, estas pautas de gobernanza buscan potenciar atributos urbanos, con el fin de atraer capitales (De Mattos, 2008). Al respecto, Hackworth (2007) desestima entender el empresarialismo urbano desde una óptica netamente local, ya que los modelos de gobernanza urbana son consecuencia de ajustes institucionales y económicos que han asumido los gobiernos nacionales adscritos a consensos internacionales, como el de Washington. En este sentido, las prácticas compelidas por gobiernos locales son parte de una estrategia -en la acepción usada por Bourdieu (2012) - de conjuntos de acciones de largo o mediano plazo, que no son necesariamente planteadas como tales y que pretenden llegar al origen de la práctica social. Esta noción de estrategia cobra sentido cuando se considera el papel del Estado como agente activo en los procesos de reestructuración urbana. De hecho, el Estado posee estrategias y emite prácticas que, a través de políticas urbanas, definen dónde y cómo invertir, desinvertir, regular o desregular (López, Gasic \& Mesa, 2012).

El urbanismo proempresarial no solo se sostiene en prácticas y estrategias directas para definir la inversión de capital. También se sustenta en discursos más o menos públicos sobre el mejoramiento de las ciudades, el aprovechamiento de oportunidades y el resurgimiento urbano (Casellas \& Poli, 2013), lo que implica tipificar las futuras zonas objeto de intervención como lugares obsoletos y degradados que requieren inversión tanto privada como pública (Weber, 2002), destinada a 
reposicionar las ciudades con un cariz atractivo para la inversión de capital dentro del mapa global. Al respecto, Harvey (1989) especifica las dimensiones en las que se manifiestan estas estrategias proempresariales: la explotación de ventajas competitivas y la atracción de actividades para dinamizar la economía e impulsar el consumo y la adquisición de funciones de control. Las prácticas proempresariales urbanas también contemplan la formación de alianzas público-privadas para la gestión urbana, la planeación, la puesta en marcha de proyectos urbanos, y la promoción de espacios potencialmente rentables para la inversión a través del establecimiento y fragmentación de zonas dentro del espacio urbano (Casellas, 2003; 2005; Hackworth, 2007; Shin, 2009).

En el contexto chileno, López et al. (2012) establecen la existencia de cuatro prácticas proempresariales de los gobiernos locales: i) la fragmentación de la planificación urbana, lo que implica una reducción de la escala de planificación y el establecimiento de zonas proclives a la inversión a través de modificaciones puntuales de los instrumentos de planificación (plan regulador comunal, seccionales, límite urbano); ii) la conformación de alianzas público-privadas, es decir, la vinculación de agentes públicos con privados a través de la conformación de corporaciones de derecho privado (como la Corporación para el Desarrollo de Santiago, Cordesan, para la comuna de Santiago), que planean estratégicamente los lineamentos para la renovación urbana; iii) la promoción de lugares específicos dentro de la comuna, destinados al desarrollo inmobiliario, iniciativa sustentada discursivamente en la futura redistribución de los beneficios y la mejora de las condiciones de vida de los habitantes; y, finalmente, iv) la absorción local de riesgos, es decir, el costo político a nivel local asumido por la realización de operaciones inmobiliarias en el territorio comunal.

Según López-Morales (2013), estas prácticas propias del pro empresarialismo urbano contenidas en estrategias para la renovación urbana permiten comprender algunos procesos de gentrificación. Al respecto, cabe señalar que la emergencia de procesos de gentrificación depende más de las políticas urbanas y la forma que adquieran los instrumentos municipales de regulación de suelo, que de determinados cambios culturales en los consumidores.

\section{Gentrificación en América Latina}

López-Morales (2013) define la gentrificación como "la reestructuración espacial de un área urbana mediante la inyección de capital fijo en mercado inmobiliario y de infraestructura orientada al reemplazo de usuarios medios-bajos por usuarios de poder económico superior, en un contexto de mercantilización del suelo" (p. 32). Esta definición, desde la geografía urbana marxista, establece que la gentrificación es producto de procesos de reinversión de capital privado, centrados en la acumulación de renta de suelo (Smith, 1979; Clark, 1988; López-Morales, 2011; 2013). Se desestiman las teorías de corte más culturalista, para las cuales la principal causa de la gentrificación vendría a ser un cambio de estilo de vida de nuevos profesionales que desean habitar en el centro de la ciudad, para enfatizar el papel del diferencial de la renta del suelo. Así, desde la teoría del rent gap se explica longitudinalmente la dinámica de la gentrificación otorgando relevancia a las diferencias producidas entre 
la renta potencial del suelo y la renta capitalizada de suelo (Smith, 1979). Desde una posición similar, Tom Slater (2009) también ha criticado las teorías culturalistas, indicando que es erróneo identificar una clase media como agente gentrificador, puesto que el fortalecimiento de alianzas entre agentes urbanos públicos y privados en la realización de planes de renovación urbana contribuye a generar una diferencia potencial de beneficio.

En esta línea de análisis, Smith (1979) pone el acento sobre el proceso de depreciación del capital fijo en áreas centrales, sustentado en la tipificación negativa (estigmatización) de barrios, el abandono y/o expulsión de residentes, y la devaluación del precio de los inmuebles. En este sentido, la gentrificación sería una forma de producción neoliberal del espacio urbano, determinada por la adopción de un modelo político-económico de desarrollo que plasma sus mayores intervenciones en la producción de nuevas formas de ciudad, y donde -aunque resulte predominante la búsqueda de beneficio por parte del capital invertido- la relación entre producción y consumo (estructura y acción) es simbiótica (Smith, 2012; Peck \& Tickell, 2002).

En el contexto latinoamericano, los estudios han enfocado la gentrificación en cuatro ámbitos: políticas urbanas para la gentrificación, gentrificación y nuevos mercados inmobiliarios, resistencias a la gentrificación y gentrificación simbólica (Janoschka et al., 2013). Es importante detenerse sobre este último aspecto, puesto que hace referencia a la puesta en valor del patrimonio urbano de barrios centrales. Entre las especificidades suscitadas en los centros históricos de América Latina, cabe subrayar la expulsión de viejos residentes a través de variados mecanismos, como la creación de subsidios para transformar residencias de carácter patrimonial en negocios de interés turístico, y los cambios en la imagen de los barrios y el paisaje urbano. Esto último, consecuencia de las transformaciones de antiguos inmuebles en hoteles, restaurantes, comercio de souvenirs, boutiques de ropa vintage, etcétera (Steel \& Klaufus, 2010). Frente a esto, se hace necesario enfatizar la importancia de la transformación de los centros históricos a través de la relaciones entre la reapropiación empresarial de los comercios, la promoción de actividades culturales que ponen de moda determinados barrios y la museificación de espacios centrales con condiciones patrimoniales (Carrión, 2007; Nelle, 2009).

A través del apoyo de denominaciones patrimoniales, locales o vía Unesco, junto con la posibilidad de optar a créditos internacionales, en las ciudades latinoamericanas se ha revalorizado o reescenificado el patrimonio urbano. El resultado ha sido la identificación del potencial económico del patrimonio arquitectónico vinculado a su explotación turística, lo que ha intensificado los procesos de gentrificación (Hiernaux, 2006). Los planes de renovación urbana o de planificación estratégica liderados por gobiernos centrales han acompańado el proceso, en ocasiones de forma encubierta, ya que en algunos casos el proceso de dinamización económica de territorios degradados con potencial turístico se ha vehiculizado a través de la mejora de la infraestructura pública y/o a través de subsidios directos a nuevos comercios (Janoschka et al., 2013). Existen casos, como los barrios Bellavista e Italia en Santiago de Chile, donde inversores privados han capitalizado económicamente ciertos valores simbólicos y arquitectónicos de aquellos lugares, eufemizados bajo la 
idea de autenticidad, produciendo procesos de regeneración urbana y gentrificación a partir de la creación de nuevas centralidades destinadas al comercio, la gastronomía y el ocio (Schlack \& Turnbull, 2011).

Los análisis realizados en otras ciudades latinoamericanas como Buenos Aires (Herzer, 2008; Kanai \& Ortega Alcázar, 2009; Lacarrieu et al., 2011), Recife (Nery \& Moura de Castillo, 2008), Quito (Carrión, 2010), Salvador de Bahía (Nobre, 2002; Ormindo de Azevedo, 2009) y Montevideo (Schelotto, 2007), Cuenca y Cusco (Steel \& Klaufus, 2010), permiten establecer que los procesos de gentrificación simbólica en Latinoamérica han sido generados por alianzas público-privadas que impulsan proyectos de recuperación de zonas patrimoniales, buscando mejorar la imagen urbana de estas zonas a fin de activar espacios atractivos para la inversión privada, el turismo y los comercios ligados a sectores económicos emergentes. Los enfoques de estos estudios han estado signados por las transformaciones de los usos de los inmuebles, la revalorización de las zonas patrimoniales y la atracción de inversión privada (Jones \& Varley, 1994; Salinas, 2013).

\section{Las políticas urbanas de Valparaíso: estrategias y prácticas para la reestructuración}

De ser el puerto latinoamericano de la costa Pacífico más importante del siglo xix, diferentes hitos nacionales e internacionales llevaron a Valparaíso a un prolongado e intenso proceso de deterioro durante el siglo xx. Entre las causa históricas de este retroceso cabe destacar la apertura del canal de Panamá en 1914, que excluyó en gran medida a Valparaíso de las rutas interoceánicas. A ello se une el fuerte impacto de la crisis de la bolsa en 1929 y su afectación negativa sobre la actividad portuaria de la ciudad (Trivelli \& Nishimura, 2010). La inestabilidad económica de Chile durante la segunda parte del siglo xx agravó el deterioro. En ámbitos productivos y laborales, la descentralización y privatización de la Empresa Portuaria de Chile (Emporchi), el fortalecimiento del puerto de San Antonio, ubicado más cerca de la capital chilena, la reducción del número de trabajadores y la implementación de leyes que flexibilizaron y precarizaron la actividad laboral portuaria generaron un empobrecimiento generalizado de las condiciones de vida de la población y un alto deterioro físico de la ciudad, que se agravó durante el período dictatorial iniciado en 1973 (Aravena, 2011).

A pesar de todo lo anterior, su situación privilegiada en la costa y larga tradición como centro comercial permiten a Valparaíso mantener un peso demográfico significativo dentro de Chile. En términos metropolitanos, las comunas de Valparaíso y Viña del Mar son los centros funcionales del Área Metropolitana del Gran Valparaíso (Carroza \& Valenzuela, 2010). A diferencia del crecimiento poblacional constante de las otras comunas que componen este territorio, Valparaíso perdió población durante el decenio 1992-2002, para luego aumentarla en prácticamente 20.000 personas entre los años 2002 y 2012, hasta alcanzar casi 295.000 habitantes (cuadro1). 
CUADro 1 | Población en la Región de Valparaíso (1992-2012)

\begin{tabular}{|l|r|r|r|}
\hline \multicolumn{1}{|c|}{ COMUNAS } & $\mathbf{1 9 9 2}$ & $\mathbf{2 0 0 2}$ & 20I2 \\
\hline Valparaíso & 282.84 & 275.982 & 294.848 \\
\hline Viña del Mar & 285.454 & 286.931 & 331.399 \\
\hline Concón & 18.872 & 32.273 & 37.765 \\
\hline Quilpué & 104.203 & 128.578 & 151.52 \\
\hline Villa Alemana & 71.672 & 95.623 & 119.397 \\
\hline Total Gran Valparaíso & 763.041 & 819.387 & 934.929 \\
\hline Total Región de Valparaíso & 1.384 .336 & 1.539 .852 & 1.643 .446 \\
\hline
\end{tabular}

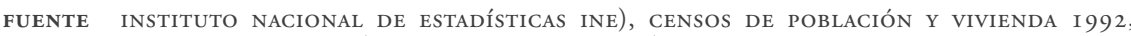
2002; Y CENSO 2012 (RESULTADOS PROVISORIOS)

El crecimiento demográfico ha tenido lugar en el marco de las políticas de renovación y promoción de la ciudad. En el año 2002, el gobierno chileno, por el Decreto Supremo 205 del Ministerio del Interior, creó "la Comisión para el desarrollo de la ciudad de Valparaíso, también denominada Comisión Plan Valparaíso, en calidad de organismo asesor del Presidente de la República, para el estudio y formulación de políticas, planes, programas, actividades y medidas tendientes al desarrollo de

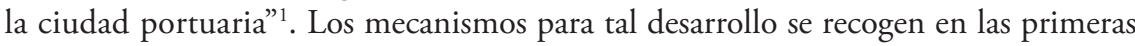
líneas del decreto, tercer Considerando:

La conveniencia de dinamizar, desarrollar, mejorar y embellecer la ciudad de Valparaíso, promoviendo y haciendo atractiva la radicación en su territorio tanto de nuevos habitantes como de inversiones, instalaciones de nuevas industrias productivas y de servicios, sin perjuicio de fortalecer el turismo, la cultura y la actividad universitaria regional (Andueza, 2010, p. 4).

El Plan Valparaíso contempló la voluntad de incentivar el crecimiento demográfico y económico de la ciudad creando condiciones de atracción tanto para residentes como para nuevos habitantes, entre los que se incluye la comunidad universitaria, inversores y turismo. En líneas generales, el plan busca fortalecer el eje ValparaísoViña del Mar como zona universitaria cobijada en los atractivos culturales y de ocio de esta conurbación, junto al fomento de actividades productivas ligadas a la hospedería y a nuevos comercios. A su vez, da cuenta de la estrategia para reactivar la economía de Valparaíso, a través de la puesta en valor del patrimonio arquitectónico y urbano de una zona de la ciudad (Consejo de Monumentos Nacionales [CMN], 2004, p. 65). Bajo esta lógica se otorga nuevas vocaciones a la ciudad-puerto, ligadas a la cultura, educación y turismo. La estrategia se ve impulsada por la denominación patrimonial, vía Unesco, de la zona del centro histórico en el año 2003.

Como se desprende del documento madre elaborado para la postulación de una zona de Valparaíso como Patrimonio de la Humanidad por la Unesco, las acciones realizadas por el Estado chileno adquieren la forma de estrategias de inversión simbólica (Bourdieu, 2012). Es decir, la intención de la postulación pretende

Véase http://legislacion-oficial.vlex.cl/vid/decreto-asesora-presidencial-valparaiso-242893358 
aumentar el capital de reconocimiento de la ciudad, promoviendo una apreciación positiva dirigida tanto hacia sus habitantes como a los futuros (y actuales) visitantes, que permita la instalación de esquemas de percepción que legitimen transformaciones de diverso orden. En el documento de postulación (CMN, 2004) se resalta la autenticidad de la ciudad -"un lugar mágico y único"-, signada por la convivencia de distintos tipos de arquitectura, el emplazamiento de viviendas en los cerros y "la vida urbana en un anfiteatro natural", junto a la presencia de artefactos como ascensores (funiculares), miradores, callejones y escaleras enclavadas en las colinas.

En la postulación a la Unesco, la promoción de la autenticidad se vuelve simbólicamente eficaz en tanto hay una organización global y hegemónica que la legitima (Unesco), pero también en cuanto es percibida por visitantes y habitantes como un valor que no necesariamente tiene existencia real, pero que sí posee efectividad en el reconocimiento que los demás le otorgan a ese valor. Bajo esa lógica, opera una suerte de consenso que reconoce un valor sobre el valor, el cual es eficaz tanto en términos de capital económico como simbólico (Bourdieu, 1997). De esta manera, la denominación de Valparaíso como patrimonio lo establece como un lugar único. $\mathrm{Al}$ respecto, Schlack y Trunbll (2011) han advertido que el reconocimiento y la valoración de la autenticidad de ciertos barrios, a propósito del impulso de procesos de renovación urbana, se articulan como atractivos y producen interés sobre el barrio, tanto para vivir como para consumir e invertir en él. Como corolario, tal interés es capitalizado económicamente por agentes privados ligados al mundo inmobiliario, y produce desplazamientos de residentes.

En esta lógica para capitalizar el reconocimiento simbólico, en Valparaíso se han creado programas y subsidios complementarios entre sí. Entre estos destaca el Programa de Recuperación y Desarrollo Urbano de Valparaíso (PRDUV), el programa Estudia en Valparaíso, el Plan de Turismo Ruмво, y los subsidios de Rehabilitación Patrimonial, tanto de la Corporación de Fomento de la Producción (Corfo), como del Ministerio de Vivienda y Urbanismo (Minvu).

Gestionado por el Gobierno Central a través de la Subsecretaría de Desarrollo Regional, el PRDUV de 2002 tiene por objetivo principal dinamizar la revitalización de la ciudad, tanto urbanística como económicamente. Para ello, el programa ha hecho uso de un crédito emanado del Banco Interamericano de Desarrollo, orientado a concentrar la inversión principalmente en la zona Unesco y sus entornos. Las líneas de acción de este programa han sido la recuperación de espacios públicos, fachadas, rehabilitación de viviendas e inmuebles emblemáticos, inmobiliario público, y promoción del turismo y la cultura. Algunas de las ideas más relevantes son sintetizadas por Andueza (2010), quien señala que el programa ha tenido por objeto la concentración de inversiones que aprovecha las ventajas competitivas de la ciudad con el fin de atraer actividades económicas y nuevos residentes.

Paralelamente a este programa, el programa Estudia en Valparaíso, creado a fines de la década del 2000, complementa la estrategia regeneradora del PRDuv. Tiene por objetivo atraer estudiantes nacionales y extranjeros para que cursen sus estudios superiores en la zona, para lo cual promociona la calidad de vida de la región, su oferta cultural y de ocio junto a la calidad de la oferta académica. Se trata de un programa público-privado gestionado por la Agencia Regional de Desarrollo 
Productivo de la Región de Valparaíso, entidad que a su vez trabaja en colaboración con las instituciones de educación superior localizadas en el eje Valparaíso-Viña del Mar. La estrategia de renovación urbana y dinamización económica vinculada a la revalorización del patrimonio arquitectónico y el turismo se complementa así con la atracción de residentes jóvenes por razones de estudio, en un contexto de expansión de la oferta de educación superior a nivel nacional (Brunner, 2009). Con un crecimiento sostenido desde el ańo 1983, en un período de diez años, entre 2001 y 2011 , se produjo un incremento aproximado de 60.000 estudiantes en la región, lo que representa un doblaje del número de estudiantes ${ }^{2}$ (figura 1). Si bien son datos a escala regional, se debe considerar que los centros universitarios se localizan casi en su totalidad dentro de la conurbación Valparaíso-Viña del Mar.

\section{FIGURA 1 Evolución estudiantes en carreras de pregrado en Chile}

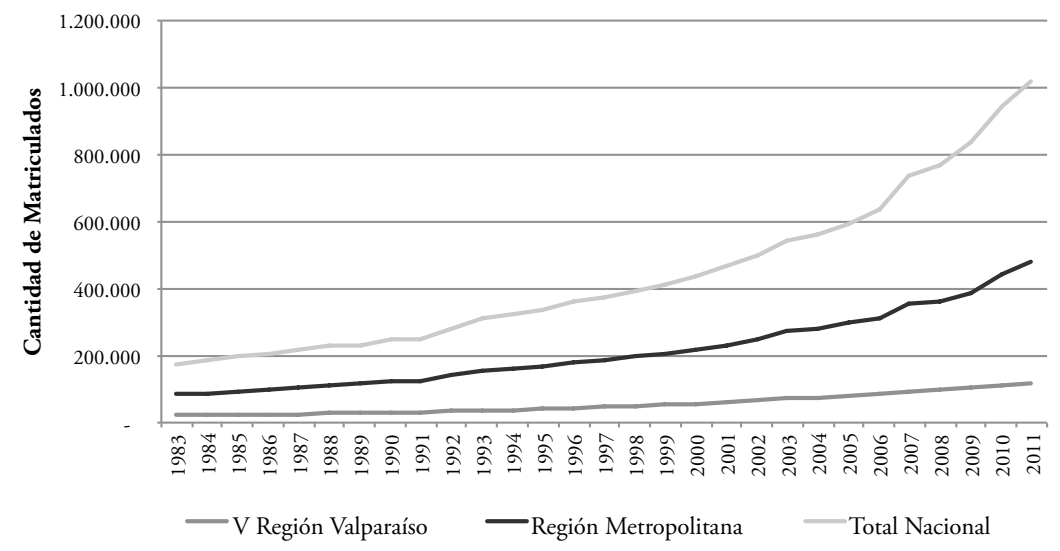

FUENTE MINISTERIO DE EDUCACIÓN, SERVICIO DE INFORMACIÓN DE EDUCACIÓN SUPERIOR (SIES) (2OII)

De carácter público-privado, el Plan Estratégico para el Destino Turístico Valparaíso RUмво tiene por objetivo diversificar la oferta turística de Valparaíso, generando nuevos polos para el desarrollo de esta actividad económica. A través de diversas estrategias de marketing, el plan estratégico de turismo desea consolidar la zona clasificada de patrimonio Unesco identificando cuatro nuevos polos de interés turístico: i) Arte y Poesía, ubicado en el entorno de la casa La Sebastiana del poeta Pablo Neruda; ii) Creamos en Polanco, en referencia a uno de los ascensores más emblemáticos de Valparaíso; iii) Ciudad Abierta al Mar, con el objetivo de promover la renovación del frente marítimo para usos náuticos y comerciales; y iv)

2 Como dato complementario, aproximadamente uno de cada cinco estudiantes de educación superior de establecimientos localizados en Valparaíso o Viña del Mar son migrantes, procedentes de otras regiones de Chile (Estudia en Valparaíso, 2011). Lamentablemente la estadística comentada no especifica la migración intrarregional, lo que nos podría dar un parámetro distinto y mucho más alto de la cantidad de estudiantes no pertenecientes a la ciudad, que estudian en el eje Valparaíso-Viña del Mar. 
Atractivos Naturales, que busca diversificar y expandir la oferta natural de la ciudad para incluir zonas localizadas fuera del límite urbano de Valparaíso.

Un elemento fundamental de la propuesta de regeneración urbana se encuentra en la financiación que ofrece la administración. El Subsidio de Rehabilitación Patrimonial Corfo, iniciado en 2001 bajo el nombre de Puerto Cultura, ofrece apoyo financiero a los emprendedores que inviertan en la compra de inmuebles catalogados como patrimoniales, para fines -totales o parciales- ligados a ámbitos productivos del turismo y la puesta en valor económico del capital cultural de Valparaíso. Por su parte, el Subsidio de Rehabilitación Patrimonial Minvu, siendo de carácter nacional, se encuentra orientado hacia quienes buscan comprar viviendas en Zonas de Conservación Histórica (zCH), definidas por el plan regulador de cada municipio. En el caso de Valparaíso, la zch contempla prácticamente todo el casco histórico, donde se localizan la zona Unesco y los nuevos polos turísticos definidos por el Plan RuмBo (figura 2).

FIGURa 2 Proyectos de renovación en el centro histórico de Valparaíso

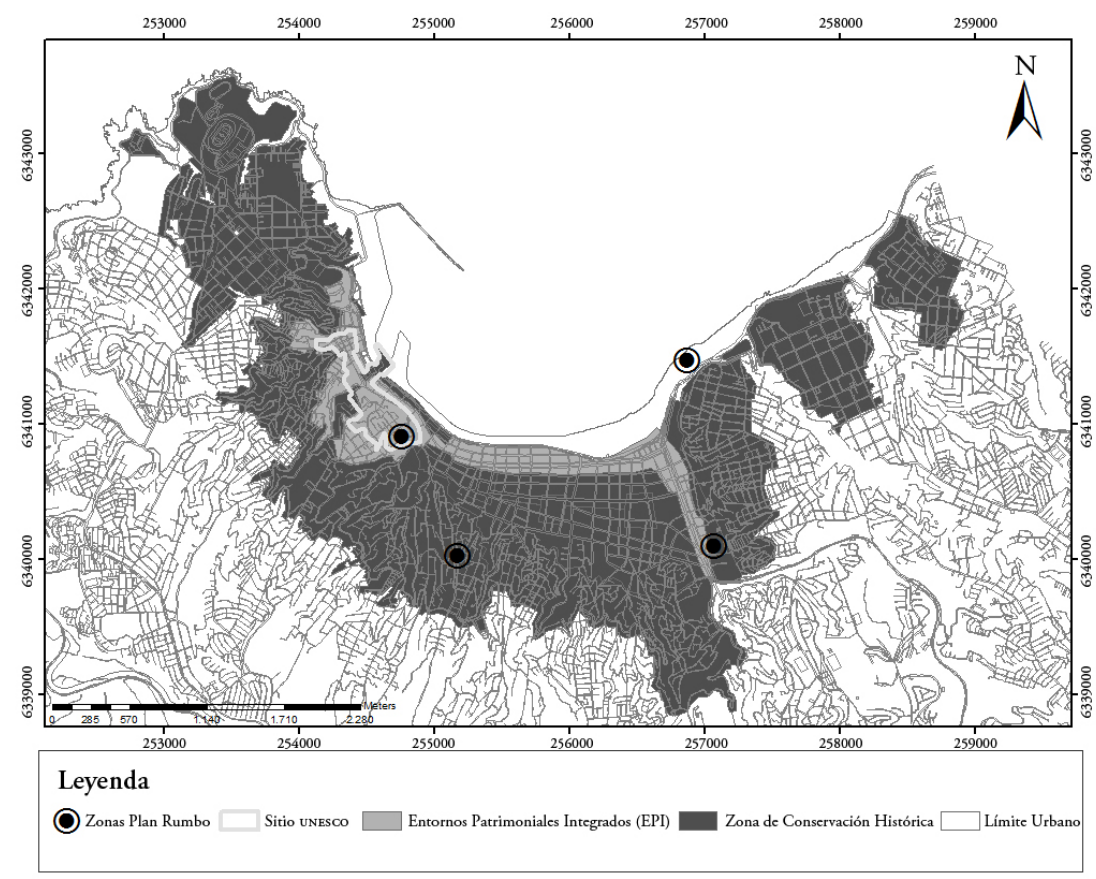

FUENTE ELABoración PROPIA A PARTIR DE PLAN REgulador DE VALPARAíso, programa de RECUPERACIÓN Y DESARROLLO URBANO DE VALPARAÍSO (PRDUV) Y PLAN RUMBO

En materia de instrumentos de planificación urbana, el Plan Regulador de Valparaíso, vigente desde 1984, fue modificado en 2005, dos años después de la denominación Unesco, para crear la denominación patrimonial local a través del establecimiento de zonas de conservación histórica (figura 2), limitar en su altura las 
nuevas edificaciones y ampliar los usos de suelo en la $\mathrm{zCH}$, favoreciendo actividades hoteleras y comerciales, principalmente vinculadas al ocio nocturno y gastronómicas $^{3}$. A pesar de estas restricciones, López et al. (2012), en su estudio sobre prácticas urbanísticas de municipios del Gran Santiago, observan que al establecerse zonas que direccionan el tipo de inversión a través de modificaciones muy puntuales a los instrumentos de planificación, se reduce la escala de planificación. En la misma línea, el establecimiento de nuevos límites en la zcH de Valparaíso posibilita un desarrollo inmobiliario de baja densidad dentro de ella, incentivando la reconversión de vivienda tipo loft u hotelería tipo boutique. A su vez, zonas como los cerros Los Placeres, Alegre, Mariposa u O'Higgins desarrollan edificación en altura a menos de diez metros de los límites establecidos en las modificaciones realizadas al Plan Regulador.

La lectura de las políticas urbanas en clave de pro empresarialismo urbano sugiere elementos para el debate. En primer lugar, del documento de postulación enviado a la Unesco (CMN, 2004) se desprende un discurso que evidencia el interés del Estado por reactivar, rehabilitar y mejorar la calidad de vida y la economía de una ciudad que posee un "valor universal". Tal como ha planteado Weber (2002), las políticas proempresariales urbanas poseen un contenido discursivo-ideológico que pretende legitimar la cualificación de espacios decaídos en potencialmente rentables, dejando en segundo plano las causas que posibilitaron la desinversión y la obsolescencia. Al respecto, Sobarzo (2009) y Guerrero (2012) enfatizan cómo la promoción de la postulación de Valparaíso como patrimonio por parte del Municipio, el Estado y algunos sectores influyentes de la sociedad civil, generó un respaldo ciudadano bajo las promesas de reactivación y producción de nuevas fuentes de empleo. Ambos autores tienden a coincidir en que estos discursos operan como dispositivos gubernamentales para legitimar la creación de espacios rentables destinados a la inversión privada.

En la misma lógica proempresarial, la búsqueda de legitimación patrimonial en la Unesco se puede interpretar como una estrategia destinada a explotar las ventajas competitivas y comparativas de la ciudad. Dicho de otra manera, se pretende reposicionar Valparaíso en el mapa global para signarla como una ciudad atractiva y competitiva para la inversión de capital (Harvey, 1989), o -dicho en términos de Bourdieu (1997) - posibilitar la agregación de valor al valor. Valparaíso entra así en la lista de ciudades como Quito, Cusco o Salvador de Bahía, todas ellas identificadas por un atractivo ligado a la autenticidad de su trama urbana y -aunque esto no en el caso de Valparaíso- un pasado colonial que persiste en lo arquitectónico.

La ampliación de las zonas de conservación histórica en el Plan Regulador Comunal actúa en el sentido propuesto por Harvey, puesto que fragmenta la escala de planificación pero ensancha el espacio en que el Estado subsidia la inversión en rehabilitación de inmuebles, tanto para vivienda como para emprendimientos comerciales u hoteleros de carácter privado. Una tónica similar adquiere la inversión estatal a través del prduv en la zona Unesco y en los Entornos Patrimoniales Integrados (EPI) (cuadro 2), puesto que evidencian la opción de mejorar la infraestructura urbana en zonas donde el Estado ha pretendido posicionar el 
patrimonio como un sector estratégico de la ciudad. La inversión privada se incentiva a través de subsidios de rehabilitación patrimonial (Corfo y Minvu) y la recuperación y posterior uso productivo de la infraestructura patrimonial, con el fin de desarrollar la oferta turística (CMN, 2004).

CuAdro 2 Inversión del Programa de Recuperación y Desarrollo Urbano de Valparaíso (PRDUV) (2009-2011)

\begin{tabular}{|l|c|}
\hline \multicolumn{1}{|c|}{ ZONA DE INVERsión } & PORCENTAJE DEL PRDU \\
\hline Zona Unesco $\left(C^{\circ}\right.$ Alegre - C ${ }^{\circ}$ Concepción - Barrio Financiero) & $46,31 \%$ \\
\hline Plaza Justicia - C ${ }^{\circ}$ Cordillera - C $C^{\circ}$ Toro & $24,91 \%$ \\
\hline Barrio Puerto - C $C^{\circ}$ Santo Domingo & $14,60 \%$ \\
\hline$C^{\circ}$ Barón - Av. Brasil - Av. Argentina & $11,51 \%$ \\
\hline Plaza Aduana - Co Artillería & $2,68 \%$ \\
\hline Total de la inversión en pesos chilenos & 7.051 .618 .995 \\
\hline
\end{tabular}

FUENTE PROGRAMA DE RECUPERACión y DESARRollo URBANO DE VALPARAÍSO (PRDUV) (2OI2)

El análisis de los agentes involucrados en el proceso, junto al estudio de los programas y subsidios analizados, permiten situar al Estado chileno como uno de los agentes urbanos más relevantes en el liderazgo de la reestructuración urbana de Valparaíso. La activación patrimonial iniciada el año 2003 se enmarca en las estrategias de reconversión posindustrial de ciudades patrimoniales latinoamericanas, las cuales han apostado por el turismo como uno de los principales polos de desarrollo (Aravena, 2006; Carrión, 2007). No obstante, la especificidad de Valparaíso radica en que la reestructuración urbana no solo se apoya en la capitalización patrimonial y el turismo, sino que también busca generar sinergias con el ámbito universitario, el cual se presenta institucionalmente como un eje clave para otorgar un cariz "rejuvenecedor" a la ciudad. El nuevo esfuerzo promocional reinterpreta la identidad de Valparaíso, en un intento de sustituir su carácter histórico de ciudad-puerto por el de ciudad-universitaria y ciudad-turística dentro del entramado metropolitano.

\section{La creación del espacio turístico y los efectos de la nominación patrimonial Unesco}

El caso de los cerros Alegre y Concepción de Valparaíso avala la interpretación de Zukin (1987), quien argumenta que los barrios caracterizados por su homogeneidad arquitectónica, centralidad, preservación histórica y/o interés patrimonial son mucho más susceptibles de caer en procesos de gentrificación que otras zonas de las ciudades. En esa dirección, la puesta en valor productivo de inmuebles con valor arquitectónico ha sido una de las estrategias de reactivación económica más utilizadas por administraciones locales en ciudades nominadas patrimonio por la Unesco (Nobre, 2002).

Se puede argumentar que en Valparaíso la aprobación de marcos normativos, el modelo de subvenciones y las alianzas público-privadas han generado espacios aptos para la inversión de capital privado y la transformación urbana. El análisis 
porcentual de los usos del suelo permite evidenciar transformaciones acaecidas en la zona Unesco y establecer que se ha producido una transformación de usos, con una progresiva pérdida del cariz residencial de la zona, para dar paso a un espacio predominantemente turístico.

Existe convergencia en considerar que los planes de renovación urbana tienen por objetivo dinamizar económicamente territorios decaídos pero con potencial arquitectónico (Hiernaux, 2006). La inversión realizada por el Estado a través del PRDUV en Valparaíso ha tendido claramente a focalizarse en la zona Unesco (cuadro 2), con más del 46\% del total de la inversión del programa por el período 2009-2011. Ello ha contribuido a una mejora significativa de lo que Harvey (1989) identifica como fondo de consumo, es decir, la infraestructura de uso público presente en el espacio.

Considerando los elementos apuntados que han facilitado la transformación de la zona Unesco, por una parte, y los datos en inversión, por otra, es posible demostrar una conjugación de la inversión pública del PRDUV con la denominación patrimonial, a la que se suman las otras gamas de subsidios públicos analizadas. Esta confluencia ha favorecido la inversión privada, facilitando los cambios en los usos de suelo de la zona. Además, cabe apuntar el régimen de tenencia como otro factor que no se debe menospreciar, ya que influye directamente en la capacidad de atracción de la inversión privada.

CUADro 3 | Régimen de tenencia de vivienda en cerros Alegre y Concepción (2002) (porcentajes)

\begin{tabular}{|l|c|c|c|}
\hline \multicolumn{1}{|c|}{ TIPO DE TENENCIA } & $\begin{array}{c}\text { ÁREA } \\
\text { METROPOLITANA } \\
\text { GRAN VALPARAÍso }\end{array}$ & VALPARAÍso & $\begin{array}{c}\text { ÁREA DE ESTUDIO } \\
\text { (CERRO ALEGRE - CERRO } \\
\text { CONCEPCIÓN) }\end{array}$ \\
\hline Propia (pagada totalmente) & 52 & 52,82 & 34,00 \\
\hline Propia (pagando a plazo) & 16 & 9,96 & 4,93 \\
\hline Arrendada & 24 & 28,15 & 55,29 \\
\hline Cedida por trabajo o servicio & 3 & 2,77 & 1,95 \\
\hline Gratuita & 5 & 6,30 & 3,83 \\
\hline Total & $\mathbf{1 0 0}$ & $\mathbf{1 0 0 , 0 0}$ & $\mathbf{1 0 0 , 0 0}$ \\
\hline
\end{tabular}

FUENTE ELABORACIÓN PROPIA A PARTIR DE CENSO DE POBLACIÓN Y VIVIENDA (INSTITUTO NACIONAL DE ESTADÍSTICAS, 2002)

El análisis de los datos del Censo de Población y Vivienda 2002 revela que el régimen de tenencia de las propiedades en los cerros Alegre y Concepción (zona Unesco) ha tendido a agilizar la transformación de la zona, dada su condición dominada por la tenencia en alquiler. Si bien la ciudad de Valparaíso y su área metropolitana tienen alrededor del $52 \%$ de la vivienda en régimen de propiedad pagada, este porcentaje cae al 34\% en el caso del área Unesco. A ello hay que ańadir que el régimen de tenencia en propiedad, pero con hipoteca, también es más elevado en el área metropolitana (16\%) y para la totalidad de la ciudad de Valparaíso (prácticamente un 10\%), que para la zona Unesco $(4,9 \%)$. Aunque no se puede afirmar que los arrendatarios 
constituyan un grupo social caracterizado por mayor vulnerabilidad ante las nuevas dinámicas de reestructuración espacial, el mayor porcentaje de viviendas en condición de alquiler en los cerros Alegre y Concepción aumenta la susceptibilidad a la expulsión. La razón es que los residentes en calidad de arrendatarios no solo pueden haber visto aumentados sus alquileres, sino que también poseen una injerencia marginal en las ventajas que aporta la renovación del barrio, puesto que quedan excluidos de la plusvalía generada en los inmuebles como resultado de la intervención pública.

De forma paralela, la naturaleza de los subsidios directos, como los de Rehabilitación Patrimonial de Corfo y del Minvu, también ha contribuido a generar una proliferación de nuevas tipologías comerciales en inmuebles destinados anteriormente a viviendas. El estudio de los cambios en el uso del suelo desde el ańo $2001 \mathrm{al}$ 2011 (cuadro 4), a través de la elaboración propia de datos aportados por el Servicio de Impuestos Internos y contrastados a través del portal oficial de la ciudad de Valparaíso, así lo evidencian. En un período de diez años, se aprecian cambios significativos en el uso de suelo. Si en 2001, año anterior a la puesta en marcha del Plan Valparaíso, el uso predominante era habitacional, complementado por comercios y algunos inmuebles adaptados a pequeñas oficinas, en el año 2011 la zona había perdido su cariz residencial, pasando a compartir este uso con comercios y pequeños hoteles. Este fenómeno de boutiquización se caracteriza por generar mayores rentabilidades del suelo urbano a través de la reconversión hacia espacios donde predominan comercios especializados, como boutiques de ropa exclusiva, restaurantes con comidas sofisticadas, galerías de arte, tiendas de souvenirs y hotelería (Carrión, 2007). En este sentido, la patrimonialización de Valparaíso ha tenido consecuencias similares a las de otras ciudades latinoamericanas denominadas Patrimonio de la Humanidad por la Unesco. Steel y Klaufus (2010) destacan que en Cusco (Perú) y Cuenca (Ecuador), las transformaciones han tenido también un impacto urbano, materializado en la instalación de bares, discotecas, boutiques y restaurantes en antiguas viviendas, principalmente destinados a la afluencia turística.

CuAdro 4 | Cambios en el uso de suelo en cerros Alegre y Concepción (20012011) (porcentajes)

\begin{tabular}{|l|r|r|c|}
\hline \multicolumn{1}{|c|}{ TIPO DE USO } & 2OOI & 2OI I & \% VARIACIÓN \\
\hline Oficina & 4 & 1 & -3 \\
\hline Educación & 2 & 1 & -1 \\
\hline Habitacional & 87 & 72 & -15 \\
\hline Hotel-Motel (hotel, motel, hostel, bed and breakfast, apart hotel) & 0 & 7 & 7 \\
\hline Almacenaje & 0 & 0 & 0 \\
\hline Otros (deporte, administración, sitio eriazo, salud) & 1 & 3 & 1 \\
\hline Culto & 1 & 1 & 0 \\
\hline Usos mixtos (hotel + comercio) & 0 & 3 & 3 \\
\hline Comercio (bares, restaurantes, comercio minorista, galerías) & 2 & 11 & 9 \\
\hline Estacionamiento & 2 & 1 & -1 \\
\hline Total & 100 & 100 & \\
\hline
\end{tabular}

FUENTE ELABORACIÓN PROPIA A PARTIR DE SERVICIO DE IMPUESTOS INTERNOS (2OOI, 2OI I) 
En Valparaíso, la revalorización del patrimonio histórico ha sido aprovechada por agentes públicos y privados como estrategia para dinamizar la economía del sector y establecer un polo marcadamente turístico en la ciudad.

El plan de marketing está relacionado a que los productos que ofrezca Valparaíso sean lo suficientemente atractivos para poder duplicar los turistas y triplicar los ingresos, esto es que los productos tengan valor para que la gente pueda gastar. Los empresarios tienen que tener una ventana y los turistas tienen que acercarse a los negocios. Por ejemplo, que en el cerro Alegre y Concepción se concentren los servicios de alojamiento, de alimentación y de compras, que sea como un Shopping patrimonial, que eleve el valor con productos de calidad. El arte y la poesía en la zona de Bellavista, con las expresiones artísticas de la ciudad (Miskovic, 2009, p.1).

La localización de los nuevos comercios y el equipamiento hotelero han tendido a concentrarse tanto dentro de los límites de la zona declarada Patrimonio de la Humanidad como en su entorno próximo e inmediato (figura 3). Más allá de los límites establecidos por la denominación patrimonial, es dentro de los límites naturales de los cerros Alegre y Concepción donde los cambios han sido manifiestos.

\section{FIGURA 3 | Localización de viviendas transformadas en hoteles y comercios en cerros Alegre y Concepción (2001-2011)}

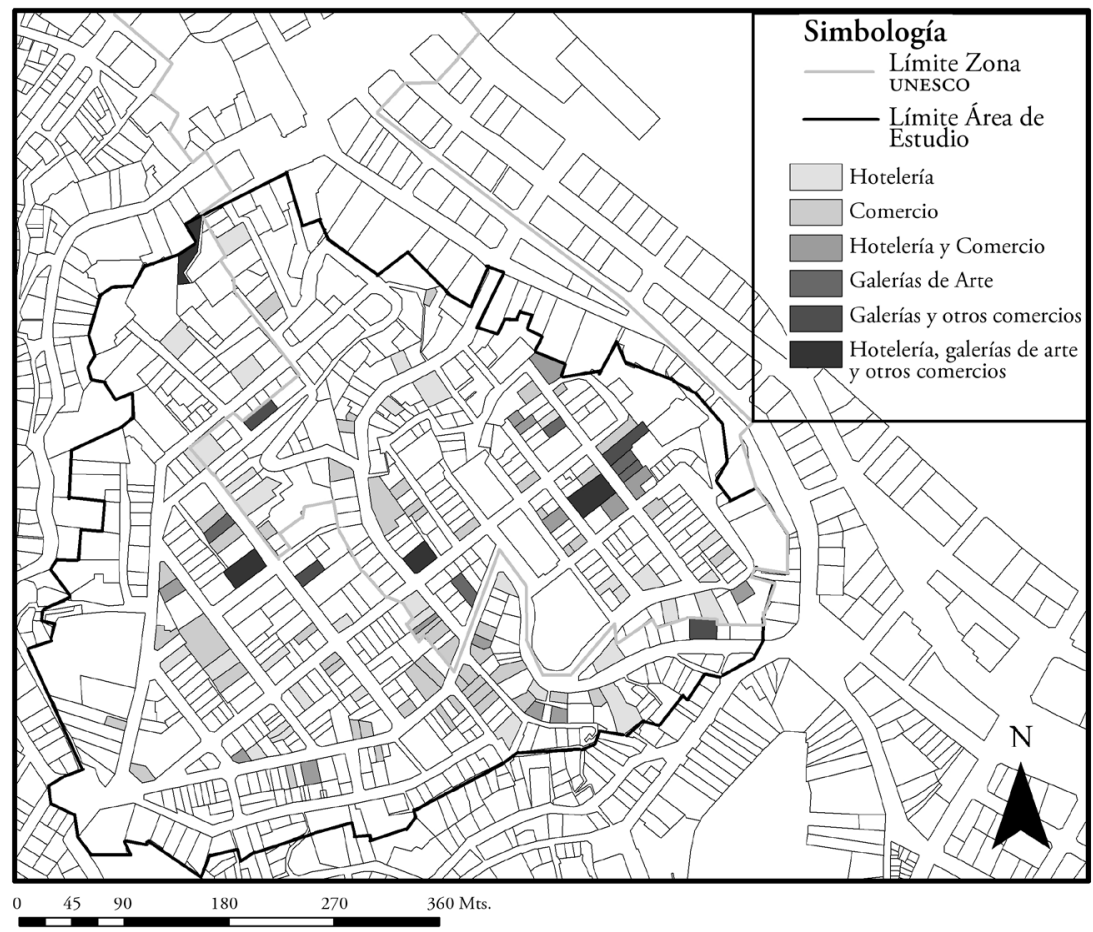

FUENTE ELABORACIÓN PROPIA A PARTIR DE SERVICIO DE IMPUESTOS INTERNOS (2OOI, 20 I I) 
La puesta en valor productivo de las características patrimoniales de estas zonas es prioritaria para funcionarios del sector público. Ahora bien, de los datos de usos del suelo trabajados en este análisis se desprenden algunos elementos que deben ser destacados. La creación de zonas turísticas afecta el tejido social y urbano de las ciudades, puesto que lo que se oferta en estos espacios está destinado básicamente a consumidores de segmentos específicos (Ormindo de Acevedo, 2009). La entrada de hotelería y usos comerciales genera pérdida de la mixtura social de barrios, dado que conlleva un uso más intenso y especializado del suelo desvinculado de las necesidades de la población local. Ello es un factor que incentiva la expulsión de habitantes y propicia la emergencia de procesos de gentrificación. Adicionalmente, tomando en cuenta el amplio crecimiento del número de turistas en Valparaíso ${ }^{4}$, se puede apuntar a su papel como agentes que influyen en las transformaciones de la zona, puesto que hacen uso de una estructura comercial hecha a su medida, produciéndose una relación simbiótica que se asocia con nuevas pautas de consumo.

\section{¿Hacia un proceso de gentrificación en Valparaíso? A modo de reflexión final}

El análisis de Valparaíso pone en evidencia el rol central del Estado chileno en los procesos de transformación urbana. Aunque algunos estudios relativos a la emergencia de gentrificación en las zonas centrales y pericentrales del área metropolitana de Santiago también han relevado el papel del sector público en los procesos de transformación urbana(véase López-Morales, 2013), en el caso de Valparaíso tal intervención ha sido guiada por una lógica diferente. En este, el papel del gobierno central, identificado como clave, activa un dispositivo -en el sentido utilizado por Agamben (2011) - patrimonial que posee legitimidad global. Ello se realiza, en primer lugar, mediante la instauración de discursos sobre la obsolescencia de la ciudad y la necesidad imperante de rehabilitación. En segundo lugar, aportando las estrategias en política pública para transformar el cariz de la ciudad, ampliando su reconocimiento identitario hacia lo turístico, lo cultural y lo universitario. Y, finalmente, actuando como soporte dinamizador de los instrumentos de planificación urbana mediante la creación de programas y políticas dentro de la institucionalidad vigente para el cumplimiento de dichas estrategias.

En el caso estudiado se evidencia el fomento estatal a la relación entre capital y cultura, puesto que en Valparaíso se recrean las condiciones culturales y económicas necesarias para que la ciudad sea reconocida como poseedora de un espacio turístico, lo que le añade una valoración positiva. Por un lado, se trabaja sobre la imagen de la ciudad mediante la exaltación de sus condiciones auténticas en materia de morfología urbana, y cómo estas pasan a convertirse en un atractivo turístico. Por otro, el Estado actúa como subsidiario a las iniciativas y emprendimientos privados que poseen sintonía con el nuevo cariz turístico de la ciudad, o -dicho de otra manera- crea programas públicos, como los subsidios patrimoniales de Corfo y Minvu, que pueden posibilitar un aumento de la renta y capturar a los inversores

4 Véase, por ejemplo, "Un 53\% aumenta el arribo de turistas a Valparaíso", El Martutino, 03 diciembre 2012, en http://www.elmartutino.cl/noticia/sociedad/un-53-aumenta-el-arribo-deturistas-valparaiso 
gracias a la utilización de un suelo ubicado dentro de una zona jerarquizada en la ciudad y, además, con estatus turístico.

Ahora bien, en términos de consecuencias urbanas y sociales, Trivelli y Nishimura (2010), en su informe sobre sustentabilidad del patrimonio urbano de Valparaíso, declaran que el impacto económico en los cerros Alegre y Concepción ha posibilitado el desplazamiento de habitantes, la instalación de comercios en inmuebles que tenían uso residencial y la llegada de nuevos residentes de un nivel socioeconómico alto. Por lo tanto, si bien aún no se cuenta con el material suficiente para indicar con autoridad la emergencia y el establecimiento de gentrificación, se ha demostrado con claridad la estrategia estatal de poner en valor productivo el patrimonio urbano de un área de la ciudad, y la existencia de un proceso en curso de reestructuración espacial de esta, que se ha materializado mediante la inyección de dineros públicos en pos de mejorar infraestructura y subsidiar inversión privada. Asimismo, nuestros datos evidencian una estructura de tenencia de la propiedad predominantemente basada en el alquiler en 2002, y una disminución del suelo usado para residencias en desmedro de usos hoteleros y comerciales en el período 2001-2011. En este sentido, la creación de un espacio turístico puede haber ejercido, o continuar ejerciendo, presiones a residentes de la zona para el desplazamiento o desplazamientos directos.

La creación de un nuevo marco estructural por parte del Estado no solo ha impulsado la inversión en territorios devaluados a través de programas y subsidios, sino que ha modificado la morfología urbana del centro histórico del puerto, creando espacios turísticos, fragmentando y jerarquizando el espacio urbano. A través de políticas urbanas y estrategias de corte proempresarial se han creado las condiciones estructurales para el desarrollo de un proceso de gentrificación que no ha sido evidenciado del todo, pero que abre líneas de investigación en esa dirección.

\section{Referencias bibliográficas}

Agamben, G. (2011). ¿Qué es un dispositivo? Revista Sociológica, 26(73), 249-264. Disponible en http://www.revistasociologica.com.mx/pdf/7310.pdf

Andueza, P. (2010). La política de activación patrimonial en Valparaíso: crítica y propuestas. Encuentro Sociedad Chilena de Políticas Públicas. Santiago: Universidad Adolfo Ibáńez. Publicado en: Mercado del Suelo Urbano Área Metropolitana de Santiago, (111), 2010. Disponible en http://bit.ly/1K6SLk6

Aravena, P. (2006). Trabajo, memoria y experiencia. Fuentes para la historia de la modernización del puerto de Valparaíso. Valparaíso: Consejo Nacional de la Cultura y las Artes / Universidad ARcis, Centro de Estudios Interculturales y del Patrimonio. Disponible en http://bit.ly/1OSV7zQ

Aravena, P. (2011). Claves para entrar al presente de Valparaíso. Cuadernos de Educación (7 abril 2011). [En línea]. Valparaíso. Disponible en http://cuadernosdeeducacion.wordpress. com/2011/04/07/claves-para-entrar-al-presente-de-valparaiso-por-pablo-aravenanunezl 
Beauregard, R. A. (1985). Politics, ideology and theories of gentrification. Journal of Urban Affairs, 7(4), 51-62. doi: 10.1111/j.1467-9906.1985.tb00094.x

Bourdieu, P. (1997). La economía de los bienes simbólicos. En P. Bourdieu, Razones prácticas. Sobre la teoría de la acción [Trad. Th. Kauf], (pp. 159-198). Barcelona: Anagrama.

Bourdieu, P. (2012). Las estrategias de la reproducción social. Buenos Aires: Siglo XxI editores.

Brunner, J. (2009). Educación superior en Chile: instituciones, mercados y politicas gubernamentales. Santiago: Editorial Universidad Diego Portales.

Carrión, F. (2007). El financiamiento de la centralidad urbana: el inicio de un debate necesario. En F. Carrión, (Ed.), Financiamientos de los centros históricos de América Latina y el Caribe. Quito: Facultad Latinoamericana de Ciencias Sociales - Flacso-Ecuador / Lincoln Institute of Land Policy.

Carrión, F. (2010). El laberinto de las centralidades históricas en América Latina. Quito: Ministerio de Cultura.

Carroza, N. \& Valenzuela, F. (2010). Transformaciones en el mercado del trabajo y expresión territorial de las desigualdades sociales: el caso del área metropolitana de Valparaíso. Revista Lider, 17(12).

Casellas, A (2003). The Barcelona Model? Agents, policies and planning dynamics in tourism development [Tesis doctoral]. Department of Urban Planning and Policy Development. Rutgers, the State University of New Jersey.

Casellas, A (2005). Aproximaciones teóricas para el análisis de la transformación urbana: Urban Regime Analysis en el contexto europeo. Documents d'Anàlisi Geogràfica, (46), 139-147. Disponible en http://ddd.uab.cat/pub/dag/02121573n46/02121573n46p139.pdf

Casellas, A. \& Poli, C. (2013). Planificación urbana e innovación: de la tecnología y la práctica profesional a la innovación social y política. Documents d'Anàlisi Geogràfica, 59(3), 435-454. doi: http://dx.doi.org/10.5565/rev/dag.101

Ciudad de Valparaíso. http://www.ciudaddevalparaiso.com/inicio/zonas_historico.php

Clark, E. (1988). The rent gap and the transformation of the built environment: Case studies in Malmo 1860-1985. Geografiska Annaler. Series B, Human Geography, 60(2), 241-254.

Consejo de Monumentos Nacionales (CMN). (2004). Postulación de Valparaíso como sitio del patrimonio mundial unesco. Segunda Serie $\mathrm{n}^{\circ}$ 7. Cuadernos del Consejo de Monumentos Nacionales. Santiago: Autor.

De Mattos, C. (2006). Modernización capitalista y transformación metropolitana en América Latina. Cinco tendencias constitutivas. En A. Geraiges, M. Arroyo \& M. Silveira (Eds.), America Latina: cidade, campo e turismo (pp. 41-63). São Paulo: Consejo Latinoamericano de Ciencias Sociales (CLACSO). Disponible en http://biblioteca. clacso.edu.ar/ar/libros/edicion/lemos/03mattos.pdf

De Mattos, C. (2008). Globalización, negocios inmobiliarios y mercantilización del desarrollo urbano. En M. Córdova (Coord.), Lo urbano en su complejidad: una lectura desde América Latina (pp. 35-62). Quito: Facultad Latinoamericana de Ciencias Sociales - Flacso-Ecuador / Ministerio de Cultura de Ecuador. Disponible en http://www. flacsoandes.edu.ec/libros/digital/40875.pdf

Estudia en Valparaíso (2011). Observatorio de la educación superior de la Región de Valparaíso. Valparaíso: Estudia en Valparaíso - IDEE Consultores. 
González, F. (2011). La disputa por la gestión de las ciudades: la reconstrucción social-urbana del campo de luchas. En G. Bailey, F. Espinoza, N. Carroza \& F. Valenzuela (Eds.), Valparaiso en tránsito: Perspectivas de una nueva sociología de la ciudad (pp. 67-82). Valparaíso: Editorial Puntángeles.

Guerrero, R. (2012). Patrimonio cultural mundial, territorio y construcción de ciudadanía. Construcción y apropiación social del patrimonio cultural de la ciudad de ValparaísoChile. Scripta Nova, Revista Electrónica de Geografia y Ciencias Sociales, 168(388). Disponible en http://www.ub.edu/geocrit/sn/sn-388.htm

Hackworth, J. (2007). The neoliberal city: governance, ideology and development in American urbanism. Ithaca, NY: Cornell University Press.

Harvey, D. (1989). From managerialism to entrepreneurialism: The transformation in urban governance in late capitalism. Geografiska Annaler. Series B, Human Geography, 71(1), 3-17.

Herzer, H. (2008). Con el corazón mirando al sur: transformaciones en el sur de la ciudad de Buenos Aires. Buenos Aires: Espacio Editorial.

Hiernaux, D. (2006). A reapropriação de bairros da Cidade do México pelas classes médias: em direção a uma gentrificação? En C. Bidou, D. Hiernaux \& H. Rivière (Eds.), De volta à cidade: dos processos de gentrificação às políticas de revitalização' dos centros urbanos (pp. 229-264). São Paulo: Annablume.

Instituto Nacional de Estadísticas (INE). (1992). Censo de Población y Vivienda Chile 1992. Santiago: Autor. Disponible en http://www.ine.cl/canales/usuarios/cedoc_online/ censos/pdf/censo_1992.pdf

Instituto Nacional de Estadísticas (INE). (2002). Censo de Población y Vivienda Chile 2002. Santiago: Autor. Disponible en http://www.ine.cl/canales/chile_estadistico/censos/ censo_poblacion_vivienda.php

Instituto Nacional de Estadísticas (INE). (2012). Resultados preliminares XviII Censo de Población y Vivienda 2012. Santiago: Autor. Disponible en http://bit.ly/1nulV2O

Janoschka, M., Sequera, J. \& Salinas, L. (2013). Gentrification in Spain and Latin America. A critical dialogue. International Journal of Urban and Regional Research, 38(4), 12341265. Doi: 10.1111/1468-2427.12030

Jones, G. \& Varley, A. (1999). The reconquest of the historic centre: Urban conservation and gentrification in Puebla, Mexico. Enviroment and Planning A, 31(9), 1547-1566. doi: $10.1068 / \mathrm{a} 311547$

Kanai, M. \& Ortega-Alcázar, I. (2009). The prospects for progressive culture-led urban regeneration in Latin America: Cases from Mexico City and Buenos Aires. International Journal of Urban and Regional Research, 33(2), 483-501. doi: 10.1111/j.14682427.2009.00865.x

Lacarrieu, M., Girola, M., Thomaz, A., Yacovino, M., Lekerman, V. \& Crovara, M. (2011). Procesos de recualificación y relegación en la ciudad de Buenos Aires. Repensando la noción de ciudad-fragmento y la despolitización de lo urbano. Revista Argumentos, 24(66), 15-34. Disponible en http://www.scielo.org.mx/scielo.php?script=sci_arttext \&pid $=$ S0187-57952011000200002

Lees, L., Slater, T. \& Wyly, E. (2007). Gentrification. Nueva York \& Londres: Routledge. 
López-Morales, E. (2011). Gentrification by ground rent dispossession: The shadows cast by large scale urban renewal in Santiago de Chile. International Journal of Urban and Regional Research, 35(2), 1-28. doi: 10.1111/j.1468-2427.2010.00961.x

López-Morales, E. (2013). Gentrificación en Chile. Aportes conceptuales y evidencias para una discusión necesaria. Revista de Geografia Norte Grande, (56), 31-52. http://dx.doi. org/10.4067/S0718-34022013000300003

López-Morales, E., Gasic, I. \& Meza, D. (2012). Urbanismo proempresarial en Chile: políticas y planificación de la producción residencial en altura en el pericentro del Gran Santiago. Revista INVI, 27(76), 75-114. http://dx.doi.org/10.4067/S071883582012000300003

Ministerio de Educación (Mineduc) / Sistema de Información de la Educación Superior (sIEs). (2011). Evolución de la matrícula de pregrado por región 1984-2011. Santiago: Autor.

Ministerio de Vivienda y Urbanismo (Minvu). Observatorio Habitacional. http://www. observatoriohabitacional.cl

Miskovic, M. (2009). Plan Rumbo: proyecto que le cambiará la cara a Valparaíso [Entrevista a Milos Miskovic, director del Departamento de Turismo de la Municipalidad de Valparaíso, por O. Aspillaga y C. Mena], El Martutino [En línea], 01 octubre 2009. Disponible en http://www.elmartutino.cl/admin/render/noticia/20587

Nelle, A. (2009). Museality in the urban context: An investigation of museality and musealization processes in three Spanish-colonial world heritage, towns. Urban Design International, 14(3), 152-171.

Nery, N. \& Moura de Castilho, C. (2008). Comunidade do Pilar e a revitalização do bairro do Recife. Possibilidades do inclusão socioespacial dos moradores ou gentrificação. Humanae, 1(2), 19-36. Disponible en http://humanae.esuda.com.br/index.php/ humanae/article/view/59

Nobre, E. (2002). Urban regeneration experiences in Brazil: Historical preservation, tourism development and gentrification in Salvador da Bahia. Urban Design International, (7), 109-124. Disponible en http://www.usp.br/fau/docentes/depprojeto/e_nobre/urban_ regeneration.pdf

Ormindo de Azevedo, P. (2009). El centro histórico de Bahía revisitado. Andamios. Revista de Investigación Social, 16(12), 95-113. Disponible en http://www.redalyc.org/articulo. oa?id=62815957005

Pacione, M. (1990). Urban problems. An applied urban analysis. Londres: Routledge.

Peck, J. \& Tickell, A. (2002). Neoliberalizing space. Antipode. A Radical Journal of Geography, 34(3), 380-404. doi: 10.1111/1467-8330.00247

Plan Director Gestión Patrimonial Valparaíso (pDgpv). (2010). Imagen-Objetivo del Plan Director de Gestión Patrimonial de Valparaíso. Levantamiento de información Fase 1. Informe Final. Valparaíso, 2010.

Plan estratégico para el destino turístico de Valparaíso "Plan Rumbo". http://www.slideshare.net/ DRKIMBO/plan-estratgico-para-el-destino-turstico-valparaso (revisado en Marzo, 2013)

Plan Regulador de la Comuna de Valparaíso. Disponible en http://www. municipalidaddevalparaiso.cl/PlanRegulador.aspx

Portal Inmobiliario. www.portalinmobiliario.cl (Revisado entre octubre 2012 y junio 2013).

Programa de Recuperación y Desarrollo Urbano de Valparaíso (PRDUV). www.prduv.cl (Revisado en marzo, 2012). 
Programa de Recuperación y Desarrollo Urbano de Valparaíso (pRDUv). Montos de inversión del PRDUv en entornos patrimoniales de la comuna de Valparaíso según sectores. [Solicitado a través de Ley de transparencia y acceso a la información pública chilena, $\mathrm{n}^{\circ} 20.288$ ].

Programa Estudia en Valparaíso. http://www.estudiaenvalparaiso.cl (Revisado en enero, 2013).

Rodríguez, P. (2007). Mecanismos financieros aplicados en la rehabilitación del centro histórico de La Habana. En F. Carrión (Ed.), Financiamientos de los centros históricos de América Latina y el Caribe (pp. 391-402), Quito: Facultad Latinoamericana de Ciencias Sociales - Flacso-Ecuador / Lincoln Institute of Land Policy.

Salinas, L. (2013). Gentrificación en la ciudad latinoamericana. El caso de Buenos Aires y Ciudad de México. Geographos [Revista digital], 4(44), 281-305. Disponible en http:// web.ua.es/es/revista-geographos-giecryal/documentos/luis-salinas.pdf?noCache= 1363271617297

Sandroni, P. (2006). Renovación urbana y gentrificación: evaluación de algunos impactos demográficos, sociales y económicos de la operación urbana Faría Lima en la ciudad de San Pablo. Revista Medio Ambiente y Urbanización, 65(1), 109-110. Disponible en http://www.ingentaconnect.com/content/iieal/meda/2006/00000065/00000001/ art00007

Schelotto, S. (2007). Breves trazos sobre el financiamiento del centro histórico de Montevideo. En F. Carrión (Ed.), Financiamientos de los centros históricos de América Latina y el Caribe (pp. 381-390). Quito: Facultad Latinoamericana de Ciencias Sociales - FlacsoEcuador / Lincoln Institute of Land Policy.

Schlack, E. \& Turnbull, N. (2011). Capitalizando lugares auténticos: artistas y emprendimientos en la regeneración urbana. ARQ, (79), 28-42. http://dx.doi.org/10.4067/S071769962011000300005

Servicio de Impuestos Internos (sII). (2001). Rol de cobro no agrícola 2001. Comuna de Valparaíso, Chile.

Servicio de Impuestos Internos (sII). (2011). Rol de cobro no agrícola 2011. Comuna de Valparaíso, Chile.

Servicio de Impuestos Internos (sII). (2013). Valores Uf. http://www.sii.cl/pagina/valores/uf/ uf2013.htm (revisado en junio, 2013).

Shin, H. (2009). Residential redevelopment and the entrepreneurial local state: The implications of Beijing's shifting emphasis on urban redevelopment policies. Urban Studies, 46(13), 2815-2839. doi: 10.1177/0042098009345540

Slater, T. (2009). Missing Marcuse: On gentrification and displacement city. СITY, 13(2), 292311. doi: 10.1080/13604810902982250

Smith, N. (1979). Toward a theory of gentrification: A back to the city movement by capital, not people. Journal of the American Planning Association, 45(4), 538-548. doi: 10.1080/01944367908977002

Smith, N. (2012). La nueva frontera urbana. Ciudad revanchista y gentrificación. Madrid: Traficante de Sueños.

Sobarzo, M. (2009). Gubernamentalidad patrimonial. En P. Aravena \& M. Sobarzo (Eds.), Valparaíso: patrimonio, mercado y gobierno. Concepción: Ediciones Escaparate. 
Steel, G. \& Klaufus, C. (2010). Displacement by/for development in two Andean cities. Artículo presentado en el 2010 Congress of Latin American Studies Association, Toronto, Canadá. Disponible en http://lasa.international.pitt.edu/members/congress-papers/ lasa2010/files/1998.pdf

Trivelli, P. \& Nishimura. Y. (2010). The sustainability of urban heritage preservation: Interventions to support economic and residential investments in urban heritage areas of Latin America and the Caribbean (RG-T1620). Case study Valparaiso. Santiago: Trivelli y Cia. Disponible en http://www.subdere.gov.cl/sites/default/files/documentos/ articles-81138_archivo_fuente.pdf

Weber, R. (2002). Extracting value from the city: neoliberalism and urban redevelopment. Antipode, 34(3), 519-540. doi: 10.1111/1467-8330.00253

Zukin, S. (1987). Gentrification: Culture and capital in the urban core. Annual Review of Sociology, (13), 129-147. doi: 10.1146/annurev.so.13.080187.001021 